\title{
DISTRIBUTION OF ZEROS OF SEQUENCES OF POLYNOMIALS OF UNBOUNDED DEGREE
}

\author{
ALAN F. KAY
}

With any infinite sequence of polynomials $\left\{f_{n}(z)\right\}$, where $f_{n}(z)$ is of degree $n$, and no two polynomials have the same degree, we associate an open set $R$ of the complex $z$ plane defined in terms of the behavior of the moduli $\left|f_{n}(z)\right|$ for large $n$. Precise definitions are given in the sequel. A connected component $R_{0}$ of $R$ is assumed to contain a point $\eta$, which we take to be the origin, such that $\left|f_{n}(\eta)\right|$, when compared asymptotically to sup $\left|f_{n}(z)\right|$ in $R_{0}$, is not too small. (See (13) and (14)). Theorem 1 ther shows that the proportion of zeros of $f_{n}(z)$ in any neighborhood of any finite boundary point of $R_{0}$ is bounded away from zero for some subsequence of the given sequence. Moreover, the number of zeros of $f_{n}(z)$ in any bounded, closed subset of $R_{0}$ is $o(n)$ for the whole sequence. These results are a generalization of an unpublished theorem of the author's thesis. $R_{0}$ is called a flat region of the sequence. Theorems 2 and 3 consider the geometry of $R_{0}$. Theorem 2 shows that $R_{0}$ cannot contain an infinite, open sector if it has a finite boundary point. The immediate interest of Theorem 2 is contained in Example (6), where sectorwise distribution of zeros is proved for a sequence of partial sums of a Taylor's series with one "big" coefficient occurring anywhere except in the earliest terms. In the corresponding theorem of Erdös and Turán $[4 ; 5]$, while a stronger type of distribution is proved, the last coefficient of each partial sum must be large. Similarly in Dvoretsky $[2 ; 3]$ the last or next to last coefficient must be large. Theorem 3 shows that any of a wide class of regions is a flat region for some sequence. Theorem 4 is the weak analogue of Theorem 1 when $f_{n}(z)$ is analytic and not necessarily a polynomial. Other examples are given discussing the converse of Theorem 1 and modifications in its hypothesis and conclusions.

Apart from one application each of Jensen's Theorem and the Fundamental Theorem of Normal Families, the proofs depend almost entirely on elementary inequalities and simple majorizations. Theorem 1 may be looked upon as a generalization, both in its statement and method of proof, of the well-known theorem of Jentzsch on the angular distribution of zeros of partial sums of Taylor's Series. 1954.

Received by the editors December 29, 1952 and, in revised form, November 1 , 
LEMMA 1. Let $\left\{f_{n}(z)\right\}$ be a sequence of functions analytic in the closure $\bar{R}_{1}$ of a region $R_{1}$ of the complex $z$-plane such that for some real sequence $\left\{k_{n}\right\}$

$$
\max \log \left|f_{n}(z) / f_{n}(0)\right|=o\left(k_{n}\right), \quad z \in \bar{R}_{1} .
$$

Let the point $q$ and the origin $O$ belong to $R_{1}$. Then for every region $R_{2}$ containing $O$ and $q$ such that $\bar{R}_{2} \subset R_{1}\left(\bar{R}_{2}\right.$ denotes the closure of $\left.R_{2}\right)$ the number of zeros of $f_{n}(z)$ in $R_{2}$ is $o\left(k_{n}\right)$.

Proof. Map some simply-connected closed subset of $R_{1}$ containing $\bar{R}_{2}$ by a schlicht function $z=h(w), h(0)=0$, onto the unit $w$ circle including its boundary. If $N_{n}(r)$ is the number of zeros of $F_{n}(w)$ $=f_{n}(h(w))$ in the circle $|w|=r<1$, then by Jensen's Theorem

$$
\int_{0}^{1} \frac{N_{n}(x)}{x} d x=\frac{1}{2 \pi} \int_{0}^{2 \pi} \log \left|\frac{F_{n}\left(e^{i \theta}\right)}{F_{n}(0)}\right| d \theta .
$$

On the one hand

$$
N_{n}(r) \log \frac{1}{r} \leqq \int_{r}^{1} \frac{N_{n}(x)}{x} d x \leqq \int_{0}^{1} \frac{N_{n}(x) d x}{x} .
$$

On the other hand

$$
\frac{1}{2 \pi} \int_{0}^{2 \pi} \log \left|\frac{F_{n}\left(e^{i \theta}\right)}{F_{n}(0)}\right| d \theta \leqq \sup _{z \in R_{1}} \log \left|\frac{f_{n}(z)}{f_{n}(0)}\right| .
$$

Thus

$$
N_{n}(r)=o\left(k_{n}\right) .
$$

Now choose $r$ so close to 1 that the image of $|w|<r$ in the $z$-plane contains $R_{2} . N_{n}(r)$ is not less than the number of zeros of $f_{n}(z)$ in $R_{2}$ so the result follows from (5).

LEMMA 2. Let $\left\{f_{n}(z)\right\}$ be a sequence of functions analytic in a bounded region $R_{1}$ with $f_{n}(0)=1$. Let $a_{1 n}, \cdots, a_{v n}$ be all the zeros of $f_{n}(z)$ in a region $R_{2}$ such that $\bar{R}_{2} \subset R_{1}$. Let $v=o\left(k_{n}\right)$ where $k_{n} \rightarrow \infty$ as $n \rightarrow \infty$, and let

$$
\limsup _{n \rightarrow \infty} \sup _{z \in \bar{R}_{1}}\left(1 / k_{n}\right) \log \left|f_{n}(z)\right|=0 ;
$$

then, if $M>0$,

$$
\limsup \sup _{n \rightarrow \infty}\left(1 / k_{n}\right) \log \prod_{i}^{0}\left|1-z / a_{i n}\right| \leqq 0 .
$$

Proof. If the lemma were false, there would exist a subsequence of 
$\left\{f_{n}(z)\right\}$ (for simplicity, not distinguished in notation) and an $\epsilon>0$ such that

$$
\prod_{1}^{0}\left|1-\frac{z_{n}}{a_{i n}}\right|>e^{e k_{n}}
$$

where $\left|z_{n}\right| \leqq M$. Let $\delta$ be a positive lower bound for the distance from $R_{2}$ to $C\left(R_{1}\right)$. [C $\left(R_{1}\right)$ denotes the complement of $R_{1}$.] Let $D$ be an upper bound for all $|z|$ with $z$ in $R_{2}$. Then if $z$ is exterior to $R_{1}$,

$$
\left|\left(a_{i n}-z\right) /\left(a_{i n}-z_{n}\right)\right|>e^{A},
$$

where $A=\log (\delta /(M+D))$. By (8) and (9), exterior to $R_{1}$,

$$
\prod_{1}^{v}\left|1-\frac{z}{a_{i n}}\right|>\exp \left(A v+\epsilon k_{n}\right) \text {. }
$$

Since $v=o\left(k_{n}\right)$,

$$
\limsup _{n \rightarrow \infty} \inf _{z \in C\left(R_{1}\right)}\left(1 / k_{n}\right) \log \prod_{1}^{v}\left|1-\frac{z}{a_{i n}}\right|>0 .
$$

By the maximum principle

$$
\sup _{z \in \bar{R}_{1}-R_{1}}\left(1 / k_{n}\right) \log \left|\frac{f_{n}(z)}{\prod_{1}^{v}\left(1-z / a_{i n}\right)}\right| \geqq 0 .
$$

Addition of (11) and (12) contradicts (6).

Definition. Let $\left\{f_{n}(z)\right\}$ be a sequence of polynomials of degree $n$ with no two of the same degree. Let $R$ be defined as a subset of the $z$-plane by: $\zeta \in R \leftrightarrow$ for some positive $\beta$

$$
\limsup _{n \rightarrow \infty} \sup _{|z-\zeta| \leqq \beta}(1 / n) \log \left|f_{n}(z)\right| \leqq 0 .
$$

$R$ is trivially open. If for some $\eta \in R$

$$
\lim _{n}(1 / n) \log \left|f_{n}(\eta)\right|=0,
$$

the connected component $R_{0}$ of $R$ containing $\eta$ will be called a flat region of the sequence $\left\{f_{n}(z)\right\}$. Without loss in generality, we assume here and in the sequel that $\eta=0$.

THEOREM 1. If a sequence of polynomials $\left\{f_{n}(z)\right\}$ has a flat region $R_{0}$, then the proportion $\mu$ of zeros of $f_{n}(z)$ lying in any neighborhood $\mathcal{O}$ of any finite boundary point $s$ of $R_{0}$ satisfies 


$$
\limsup _{n \rightarrow \infty} \mu=\mu(n, \mathcal{O})>0 .
$$

Proof. We may obviously assume that $\mathcal{O}$ is a circle about $s$ not containing the origin. Let $q \in R_{0} \cap \mathcal{O}$. Let $t \in \mathcal{O} \cap C(R)[C(R)$ denotes the complement of $R$ ]. Let $\mathcal{O}^{\prime}$ be a circle about $s$, smaller than $\mathcal{O}$ so that $\rho$, the min. distance of $\mathcal{O}^{\prime}$ to $C(\mathcal{O})$, is positive and so that $q \in \mathcal{O}^{\prime}$, and $t \in \mathcal{O}^{\prime}$. Let $R_{1}$ be a bounded, simply connected subregion of $R_{0}$ such that $0, q \in R_{1}$ and $\bar{R}_{1} \subset R$. Then it follows from (13) that

$$
\limsup _{n \rightarrow \infty} \sup _{z \in \bar{R}_{1}}(1 / n) \log \left|f_{n}(z)\right| \leqq 0 .
$$

From (14) we find that equality holds in (16) and that

$$
\lim _{n \rightarrow \infty} \sup _{z \in \bar{R}_{1}}(1 / n) \log \left|f_{n}(z) / f_{n}(0)\right|=0 .
$$

Let $R_{2}$ be a simply-connected region containing 0 and $q$ and such that $\bar{R}_{2} \subset R_{1}$. Applying Lemma 1 with $k_{n}=n$ implies that the number $v=v(n)$ of zeros of $f_{n}(z)$ in $R_{2}$ satisfies

$$
v=o(n) \text {. }
$$

Now let us order the zeros of $f_{n}(z)$ (multiple zeros listed multiply) so that $a_{1 n}, \cdots, a_{v n}$ are all the zeros of $f_{n}(z)$ in $R_{2}$. Let $b_{1 n}, \cdots, b_{w n}$ be all the zeros of $f_{n}(z)$ in $\mathcal{O}^{\prime} \cap C\left(R_{\mathbf{2}}\right)$ and let $c_{1 n}, \cdots, c_{x n}$ be the remaining zeros of $f_{n}(z)$. Suppose the theorem false. Then $w=o(n)$. Now, if $z \in C\left(R_{1}\right)$,

$$
\prod_{1}^{0}\left|1-z / a_{i n}\right|>(\delta / D)^{v} .
$$

Here $\delta$ is a positive lower bound for the distance between a point in $R_{2}$ and a point in $C\left(R_{1}\right)$, and $D$ is an upper bound for the absolute value of points in $R_{2}$. Let

$$
\begin{aligned}
g_{n}(z) & =\left(f_{n}(z) / f_{n}(0)\right) / \prod_{1}^{v}\left(1-z / a_{i n}\right) \\
& =\prod_{1}^{w}\left(1-z / b_{i n}\right) \prod_{1}^{x}\left(1-z / c_{j n}\right) .
\end{aligned}
$$

Now by (17), (18), (19) and the maximum principle,

$$
\max \left|g_{n}(z)\right|=e^{o(n)}, \quad z \in \text { the boundary of } R_{1} .
$$

Since $g_{n}(z)$ is analytic, (21) holds for all $z$ in $R_{1}$. We see from (20) that, for any value of $z$ 


$$
\left|g_{n}(z)\right| \leqq(1+|z| / \epsilon)^{n},
$$

where $\epsilon$ is a positive lower bound for the absolute value of points in $C\left(R_{2}\right)$. Let

$$
h_{n}(z)=\prod_{1}^{x}\left(1-z / c_{j n}\right)
$$

If $z$ is exterior to $\mathcal{O}$,

$$
\left(\frac{\rho}{M^{\prime}}\right)^{w} \leqq \prod_{1}^{w}\left|1-\frac{z}{b_{i n}}\right| \leqq\left(1+\frac{|z|}{m^{\prime}}\right)^{w},
$$

where $M^{\prime}=\sup |z|, z \in \mathcal{O}^{\prime}$; and $m^{\prime}=\inf |z|, z \in \mathcal{O}^{\prime}$. Therefore, if $z$ is exterior to $\mathcal{O}$,

$$
\left|h_{n}(z)\right| \leqq(1+|z| / \epsilon)^{n}\left(M^{\prime} / \rho\right)^{w},
$$

and by the maximum principle, interior to $\mathcal{O}$

$$
\left|h_{n}(z)\right| \leqq(1+M / \epsilon)^{n}\left(M^{\prime} / \rho\right)^{w},
$$

where $M=\sup |z|, z \in \mathcal{O}$. Whereas by (21) and (24)

$$
\sup \left|h_{n}(z)\right|=e^{o(n)}, \quad z \in R_{1} \cap C(\mathcal{O}) .
$$

Let $\alpha$ be a small positive number so that if $|z-t| \leqq 2 \alpha, z \in \mathcal{O}^{\prime}$. Now since $t$ is not in $R$

$$
\limsup _{n \rightarrow \infty} \sup _{|z-t| \leqq \alpha}(1 / n) \log \left|f_{n}(z) / f_{n}(0)\right|>0 .
$$

For a subsequence, which in view of (15) we may assume to be the original sequence,

$$
\lim _{n \rightarrow \infty} \sup _{|z-t| \leqq \alpha}(1 / n) \log \left|f_{n}(z) / f_{n}(0)\right|>0 .
$$

Since $v=o(n)$, in view of (17) we may apply Lemma 2 with $k_{n}=n$, so that by (7) and (29)

(30) $\liminf _{n \rightarrow \infty} \sup _{|z-t| \leqq \alpha}(1 / n)\left\{\log \left|f_{n}(z) / f_{n}(0)\right|-\log \prod_{1}^{0}\left|1-z / a_{i n}\right|\right\}>0$.

Also

$$
\sup _{|z-t| \leqq \alpha} \prod_{1}^{w}\left|1-z / b_{j n}\right| \leqq\left(2 M^{\prime} / m^{\prime}\right)^{w}=e^{o(n)}
$$

and so 


$$
\liminf _{n \rightarrow \infty} \sup _{|z-t| \leqq \alpha}(1 / n) \log \left|h_{n}(z)\right|>0 .
$$

Now $h_{n}(z)$ has no zeros interior to $R_{2} \cup \mathcal{O}^{\prime}$ and $h_{n}(0)=1$. By choice of branch such that $\left(h_{n}(0)\right)^{1 / n}=1$, we may define a single-valued function $F_{n}(z)=\left(h_{n}(z)\right)^{1 / n}$ analytic in $R_{2} \cup \mathcal{O}^{\prime}$ such that

$$
F_{n}(0)=1 \text {. }
$$

Moreover by (25) and (26) we see that in $R_{2} \cup \mathcal{O}^{\prime}\left|F_{n}(z)\right|$ is bounded uniformly in $n$. By the fundamental theorem of normal families, there exists a subsequence converging uniformly to an analytic limit $F(z)$ in $T$, a closed, simply-connected subset of $R_{\mathbf{2}} \cup \mathcal{O}^{\prime}$ containing the circles $|z| \leqq \epsilon / 2$ and $|z-t| \leqq \alpha$. By (33),

$$
F(0)=1 \text {. }
$$

By (27),

$$
\max |F(z)| \leqq 1,
$$$$
z \in T \cap C(\mathcal{O})
$$

and since $F(z)$ is analytic, $F(z) \equiv 1$ in $T$. But by (32) at some point in the circle $|z-t| \leqq \alpha,|F(z)|>1$. Since this point lies in $T$, we have the contradiction which establishes the theorem.

Theorem 2. Let $R$ be a flat region of a sequence of polynomials $\left\{f_{n}(z)\right\}$. If $R$ has a finite boundary point $s$, then $R$ cannot contain any infinite sector.

Proof. Let $C$ be a circle with center $\zeta$ and radius $a \cdot|\zeta|, 0<a<1$. We shall show that if $|\zeta|$ is sufficiently large, $C$ cannot lie wholly in $R$. This will establish the theorem. Let $\epsilon$ be positive. Let $a_{1 n}, \cdots$, $a_{k n}$ be all the zeros of $f_{n}(z)$ in the circle $|z-s|<\epsilon$. Let $a_{k+1, n}, \cdots, a_{m n}$ be all the zeros, not already listed, and lying exterior to the circle $C^{\prime}$ of radius $2 a|\zeta|$ and center $\zeta$. Let $a_{m+1, n}, \cdots, a_{n n}$ be the remaining zeros. We have

$$
\inf \prod_{1}^{k}\left|1-\frac{z}{a_{i n}}\right| \geqq\left[\frac{|\zeta|(1-a)}{|s|+\epsilon}-1\right]^{k}, \quad z \in C,
$$

if $(1-a)|\zeta|>|s|+\epsilon$. Also

$$
\inf \prod_{k+1}^{m}\left|1-\frac{z}{a_{i n}}\right| \geqq\left(\frac{a}{1+2 a}\right)^{m-k}, \quad z \in C .
$$

The minimum modulus property of the Tschebyscheff polynomials for a circle yields 


$$
\max _{|w| \leqq 1} \prod_{j=m+1}^{n}\left|w-w_{j}\right| \geqq 1
$$

for any set of points $w_{j}$. Thus if $w=(z-\zeta) / a \zeta$,

$$
\begin{aligned}
\sup _{z \in C} \prod_{m+1}^{n}\left|1-\frac{z}{a_{i n}}\right| & =\max _{|w|=1}\left\{\frac{|a \zeta|^{n-m} \prod_{m+1}^{n}\left|\left(a_{i n}-\zeta\right) / a \zeta-w\right|}{\prod_{m+1}^{n}\left|a_{i n}\right|}\right\} \\
& \geqq\left(\frac{a}{1+2 a}\right)^{n-m} .
\end{aligned}
$$

Combining (36), (37), and (38):

$$
\sup _{z \in C} \prod_{1}^{n}\left|1-\frac{z}{a_{i n}}\right| \geqq\left(\frac{a}{1+2 a}\right)^{n-k}\left[\frac{|\zeta|(1-a)}{|s|+\epsilon}-1\right]^{k} .
$$

By Theorem $1, \lim \sup _{n \rightarrow \infty}(k / n)>0$ as $n \rightarrow \infty$; hence if $|\zeta|$ is sufficiently large

$$
\underset{n \rightarrow \infty}{\limsup } \sup _{z \in C}(1 / n) \log \left|f_{n}(z) / f_{n}(0)\right|>0 .
$$

$C$, therefore, cannot be contained in $R$. Since $a$ is arbitrarily small, no infinite sector can be contained in $R$.

It is interesting to note that if $R$ does contain $C$ for fixed $|\zeta|$ $>2(|s|+\epsilon) /(1-a),(39)$ gives an upper bound for $k / n$, the proportion of zeros in a small circle about $s$. Thus from the geometry of $R$ and no other information about the original sequence of polynomials we can obtain information about the distribution of the zeros near the boundary of $R$.

A flat region can have essentially any shape. In particular:

TheOREM 3. Given a region $R^{0}$ bounded by an analytic Jordan curve, there exists a sequence of nested regions $R^{m}$ whose union is $R^{0}$ and such that each $R^{m}$ is a flat region for some sequence of polynomials $f_{n}^{m}(z)$.

Proof. We use the theorem that the boundary of the region $R^{0}$ may be approximated by lemniscates bounding nested regions $R^{m}$ whose union is $R^{0}$. This is proved in [17]. Let $p_{m}(z)$ be a polynomial such that the lemniscate $\left|p_{m}(z)\right|=1$ is the boundary of $R^{m}$, and suppose its degree is $d$. Then

$$
\left|p_{m}(z)\right|<1, \quad \text { if } z \in R^{m},
$$


and if $\alpha>0$

$$
\sup \left|p_{m}(z)\right|>1, \quad\left|z-z_{0}\right|<\alpha,
$$

if $z_{0}$ is on the boundary of $R^{m}$. If we define, for all integers $k>0$,

$$
f_{n}^{m}(z)=\left[p_{m}(z)\right]^{k}+1, \quad n=d k,
$$

the theorem follows readily.

EXAMPles AND Corollaries (using the notation of the above theorems).

(1) Converse of Theorem 1. A strong converse is not true: Let $f_{n}(z)=\left(1-e^{n} z\right)\left(1-z^{n-1}\right)$. Here $f_{n}(0)=1$, only one zero of $f_{n}(z)$ lies in $|z|<1$, the remaining zeros are uniformly distributed on the unit circle. Yet, $R_{0}$ is the empty set. A partial converse is true: the proportion $\mu$ of zeros of $\left\{f_{n}(z)\right\}$ [i.e., the number of zeros divided by the degree $n$ ] in a sufficiently small neighborhood of any interior point of its flat region $R_{0}$ satisfies $\lim \mu=0$. This follows from Lemma 1 .

(2) Sectorwise distribution. If a flat region has no finite boundary point, in view of the partial converse given in example (1), the proportion $\nu$ of zeros in any bounded region is $o(1)$. Thus, by Theorem 2, if $\left\{f_{n}(z)\right\}$ has a flat region and $\nu \neq o(1)$ is the proportion of zeros of $f_{n}(z)$ in some fixed bounded region, the proportion $\epsilon$ of zeros of $f_{n}(z)$ in any sector with vertex at the origin satisfies: $\lim \sup , n \rightarrow \infty, \epsilon>0$.

(3) Strengthening the conclusion of Theorem 1. " 0 " in (15) cannot be replaced by any positive number independent of $n$. In fact, for fixed $M$ and $\epsilon$ satisfying $M>1$ and $1>\epsilon>0$, there exists $\left\{f_{n}(z)\right\}$ whose flat region is the unit circle and yet less than $\epsilon n$ zeros of $f_{n}(z)$ lie in the circle $|z|<M$ :

$$
f_{n}(z)=\left(1-z^{k}\right)\left[1-(z / M)^{n-k}\right] \quad \text { where } k=[\epsilon n]
$$

(4) $R^{0}$ not generally a flat region. One might expect that some type of "diagonal" sequence $f_{n}^{m}(z)$ in Theorem 3 would have $R^{0}$ as its flat region $R$. In view of Theorem 2 , however, this is seen to be generally impossible. How a diagonalization process breaks down is illustrated in the following example: $f_{n}^{m}(z)=1+\left[1+(z-1) / r_{m}\right]^{n}, \quad r_{m}$ real, $\lim r_{m}=\infty$ as $n \rightarrow \infty$. Here the regions $R^{m}$ are circles of radii $r_{m}$ all tangent to the line $R(z)=x=1$ at $z=1$ and lying to the left of this line. The zeros of $f_{n}^{m}(z)$ are equally spaced along the circumference of $R^{m}$. If $r_{m}=o(n)$, any point $s$ of the line $x=1$ is a limit point of zeros of $\left\{f_{n}^{m}(z)\right\}$ and no other points of the plane enjoy this property. However, the proportion of zeros of $f_{n}^{m}(z)$ in a neighborhood of $s$ is zero in the limit. The half-plane to the left of $z=1$ cannot be a flat region $R$ therefore. Examination shows that no matter how slowly $r_{m}$ grows, 
$\log \left|f_{n}(z)\right| / n=o(1)$ uniformly in any bounded region. $R$ is thus the whole plane.

(5) Complete region of uniform convergence. If the sequence $\left\{f_{n}(z)\right\}$ has a complete region $S$ of uniform convergence in the sense of Ostrowski [10], $S$ is a subregion of $R$. If $f_{n}(z)=1+z^{m}+(z / 2)^{n}$ where $m=o(n), S$ is the unit circle, $R$ is the circle $|z|<2$. If $1 / 2>\delta>0$ for $n \geqq n_{0}(\delta)$ precisely $m$ zeros lie in the ring $1-\delta \leqq|z| \leqq 1+\delta$, no zeros lie either in $|z|<1-\delta$ or in the ring $1+\delta<|z|<2-\delta, n-m$ zeros lie in $2-\delta$ $<|z|<2+\delta$.

(6) Sequences of partial sums of Taylor's series. Let $\delta$ be positive. If $\left\{f_{n}(z)\right\}$ is a subsequence of the partial sums of Taylor's series

$$
1+\sum_{1}^{\infty} a_{i} z^{i}
$$

with a finite circle of convergence such that for some $k=k(n), n \geqq k>\delta n$, the inequality, $\left|a_{k}\right|>\delta^{k}$, holds, then if $\epsilon n$ is the number of zeros of $f_{n}(z)$ in a fixed infinite sector $\xi$, with vertex at the origin, lim sup, $n \rightarrow \infty, \epsilon>0$. (Compare Dvoretsky $[2 ; 3]$.)

Proof. The sequence $\left\{f_{n}(z)\right\}$ has a flat region $R$ containing the circle of convergence in view of Example 5. Since if $r$ is sufficiently large

$$
\max _{|z|=r}\left|f_{n}(z)\right|>(\delta r)^{k} \neq e^{o(n)},
$$

$R$ contains one finite boundary point. The result then follows from Theorems 1 and 2.

(7) A region $U$ related to the flat region. Let $U$ be defined by: $\zeta \in U \leftrightarrow$ there exists $\beta>0$ such that

$$
\sup \left|f_{n}^{\prime}(z) / f_{n}(z)\right|=o(n), \quad|z-\zeta|<\beta .
$$

If the origin is in $U$ and $U_{0}$ is the connected component of $U$ containing the origin, then $U_{0}$ is contained in a flat region $R_{0}$. Moreover, if $\zeta \in R_{0}$ and there exists a connected subregion $V$ of $R_{0}$ containing $\zeta$ and 0 and free of all zeros of $f_{n}(z)$ for all $n>n_{0}$, then $\zeta \in U_{0}$. Every finite boundary point of $U_{0}$, therefore, is a limit point of zeros of $f_{n}(z)$. We shall actually prove a more general result:

(8) Let $R_{2}$ be a simply-connected region such that for some open set $R_{1}, \bar{R}_{2} \subset R_{1}$, and $\bar{R}_{1} \subset R_{0}$. Let $g_{n}(z)$ be the polynomial as defined by (20) obtained from $f_{n}(z)$ by factoring out all the zeros of $f_{n}(z)$ in $R_{2}$ (the normalization at the origin is not necessary). Define an open set $V$ by: $\zeta \in V \leftrightarrow$ there exists a positive $\beta$ such that

$$
\sup \left|g_{n}^{\prime}(z) / g_{n}(z)\right|=o(n), \quad|z-\zeta|<\beta .
$$


Define $W_{0}$ as the restriction to $R_{1}$ of the maximal region which (a) contains the origin and (b) is free of all zeros of $g_{n}(z)$ for all $n>n_{0}$; then we shall prove that the origin is in $V$, and the connected component $V_{0}$ of $V$ containing the origin satisfies

$$
W_{0} \subseteq V_{0} \subseteq R_{0}
$$

We note first that if $R_{2}$ is chosen as the empty set Corollary (8) reduces to Corollary (7). For the proof we require

LEMma 3. Let $\left\{f_{n}(z)\right\}$ be a sequence of functions analytic in a circle $C:|z-\zeta| \leqq r_{1}$ and let $k_{n} \rightarrow \infty$ as $n \rightarrow \infty$. If

$$
\max \left|f_{n}^{\prime}(z) / f_{n}(z)\right|=o\left(k_{n}\right), \quad z \in C,
$$

then

$$
\max \left|f_{n}(z) / f_{n}(\zeta)\right|=e^{o\left(k_{n}\right),} \quad z \in C ;
$$

and conversely, if (48) holds, and the functions $f_{n}(z)$ are not zero in $C$, (47) holds in a smaller circle, i.e., if $r^{\prime}<r$,

$$
\max \left|f_{n}^{\prime}(z) / f_{n}(z)\right|=o\left(k_{n}\right), \quad|z-\zeta| \leqq r^{\prime} .
$$

Proof. If $z$ lies in $C$

$$
\begin{aligned}
\log \left|f_{n}(z) / f_{n}(\zeta)\right| & =\mathbb{R} \int_{\zeta}^{z} f_{n}^{\prime}(t) / f_{n}(t) d t \\
& \leqq|z-\zeta| \cdot \max _{z \in C}\left|f_{n}^{\prime}(z) / f_{n}(z)\right|=o\left(k_{n}\right)
\end{aligned}
$$

and the first half of the lemma is proved. We apply a Theorem of Borel and Carathéodory (see Titchmarsh [15]) to $\log \left(f_{n}(z) / f_{n}(\zeta)\right.$ ) to obtain

$$
\max _{|z \rightarrow \zeta|=r^{\prime}}\left|f_{n}^{\prime}(z) / f_{n}(z)\right| \leqq\left[8 r /\left(r-r^{\prime}\right)^{2}\right] \max _{|z-\zeta|=r} \log \left|f_{n}(z) / f_{n}(\zeta)\right|,
$$

and the second half of the lemma follows directly.

Now suppose that $\zeta \in W_{0}$, then there exists $\beta$ such that if $|z-\zeta| \leqq \beta$, $z \in W_{0} \subseteq R_{1}$. Suppose that $\zeta$ does not belong to $V$. Then for some subsequence of $\{n\}$, which for simplicity we do not distinguish in notation, and for some positive $\delta$,

$$
\sup \left|g_{n}^{\prime}(z) / g_{n}(z)\right|>\delta n, \quad|z-\zeta|<\beta / 2 .
$$

Since $g_{n}(z)$ is free of zeros in $W_{0}$, we may define there a single-valued, analytic function, the branch of the $n$th root of $g_{n}(z)$ which is unity at the origin. By (21), we are permitted an application of the fundamental theorem of normal families leading to: 


$$
\lim _{j}\left(1 / n_{j}\right)\left(\log \left|g_{n_{j}}(z)\right|-\log \left|g_{n_{j}}(\zeta)\right|=0,\right.
$$

uniformly in $|z-\zeta| \leqq \beta$, for some subsequence $\left\{n_{j}\right\}$ of $\{n\}$. By the converse of Lemma 3, (52) contradicts (51). This implies that $\zeta \in V$ and hence $W_{0} \subseteq V$. Thus the origin is in $V$ and $W_{0} \subseteq V_{0}$.

To prove the remainder of (46), let $\zeta \in V_{0}$. Then there exists a finite chain of circles $C_{j}$ with centers $\zeta_{j}, j=1, \cdots, m$, such that $C_{j} \subset V_{0}, \zeta_{j+1} \in C_{j}$, and $\zeta_{1}=0, \zeta_{m}=\zeta$. By Lemma 3, max $\left|g_{n}(z) / g_{n}\left(\zeta_{j}\right)\right|$ $=e^{o(n)}, z \in C_{j}, j=1, \cdots, m$. Taking the product with respect to $j$ and majorizing we find

$$
\max \left|g_{n}(z)\right|=e^{o(n)}, \quad z \in C_{m} .
$$

We have that for $z \in C_{m}$

$$
\max \log \left|\frac{f_{n}(z)}{f_{n}(0)}\right| \leqq \max \log \left|g_{n}(z)\right|+\max \log \prod_{1}^{v}\left|1-\frac{z}{a_{i n}}\right| .
$$

If we set $k_{n}=n$ in Lemma 2, we see that the last term in (54) is $o(n)$. Then in view of (53) the whole right side of (54) is $o(n)$. Thus $\zeta \in R$, and $V_{0} \subseteq R_{0}$.

TheOREM 4. Let $\left\{k_{n}\right\}$ be a real sequence, $k_{n} \neq 0$. Let $\left\{f_{n}(z)\right\}$ be functions analytic in $R_{0}$, a region containing the origin. Let

$$
\lim _{n}\left(1 / k_{n}\right) \log \left|f_{n}(0)\right|=0 .
$$

Suppose that for every closed subset $A$ of $R_{0}$

$$
\limsup _{n \rightarrow \infty} \max _{z \in A}\left(1 / k_{n}\right) \log \left|f_{n}(z)\right| \leqq 0 .
$$

Suppose $\mathcal{O}^{\prime} \subset \mathcal{O}$ are two circles about $s$, a finite boundary point of $R_{0}$, such that $f_{n}(z)$ may be continued throughout $\mathcal{O}$ and

$$
\limsup _{n \rightarrow \infty} \max _{z \in \mathcal{O}}\left(1 / k_{n}\right) \log \left|f_{n}(z)\right|<\infty,
$$

and

$$
\sup _{n} \max _{z \in \mathcal{O}^{\prime}}\left(1 / k_{n}\right) \log \left|f_{n}(z)\right|>0 .
$$

Then $\mathcal{O}$ contains a zero of $f_{n}(z)$ for all $n$ sufficiently large.

The proof of Theorem 4, being similar to and simpler than that of Theorem 1, is omitted.

A comparison of Theorem 1 and the Theorems of Erdös and Turán $[4 ; 5]$ is made in a companion paper. 


\section{BIBLIOGRAPHY}

1. F. Carlson, Sur les fonctions entières, Arkiv för Matematik, Astronomi och Fysik, vol. 35A, No. 14, 1948.

2. A. Dvoretsky, On sections of power series, Ann. of Math. (1950) pp. 643-696.

3. - On the theorem of Jentzsch, Proc. Nat. Acad. Sci. U.S.A. (1949) pp. 246252.

4. P. Erdös and P. Turán, On the distribution of roots of polynomials, Ann. of Math. (1950) pp. 105-119.

5. - On a problem in the theory of uniform distribution, Nederl. Akad. Wetensch. vol. 51 (1948) pp. 1146-1154, 1262-1269.

6. J. Korevaar, The zeros of approximating polynomials and the canonical representation of an entire function, Duke Math. J. vol. 18 (1951) pp. 573-592.

7. E. Lindelöf, Sur la determination de la croissance des fonctions entières définies par un dévelopement de Taylor, Bull. Sci. Math. vol. 27 (1903) pp. 213-223.

8. E. Lindwart, Über eine Methode von Laguerre zur Bestimmung des Geschlechts einer ganzen Funktion, Inaugural Dissertation, University of Göttingen, 1914.

9. G. MacLane, Polynomials with zeros on a rectifiable Jordan curve, Duke Math. J. vol. 16 (1949) pp. 461-474.

10. A. Ostrowski, Über vollständige Gebiete gleichmässiger Konvergenz von Folgen analyticher Funktionen, Abh. Math. Sem. Hansischen Univ. vol. 1 (1922) pp. 327-350.

11. G. Polya, Über Annäherung durch Polynome deren Sämtlichen Wurzeln in einem Winkelraum fallen, Nachr. Ges. Wiss. Göttingen (1913) pp. 325-330.

12. P. Rosenbloom, Trans. Amer. Math. Soc. (forthcoming). Bull. Amer. Math. Soc. Abstracts 48-11-321 and 49-9-210.

13. G. Szegö, Über eine Eigenschaft der Exponentialreihe, Berlin Math. Ges. Sitzungsber. (1924) pp. 50-64.

14. - Über die Nullstellen von Polynomen, die in einem Kreise gleichmässig konvergieren, Berlin Math. Ges. Sitzungsber. vol. 21 (1922) pp. 59-64.

15. E. C. Titchmarsh, The theory of functions, Oxford University Press, 1950, p. 176.

16. G. Valiron, Lectures on the general theory of integral functions, Toulouse, Edouard Privat, 1923, Chap. II.

17. J. L. Walsh, Interpolation and approximation in the complex domain, p. 68, Theorem 2.

Technical Research Group 